OPEN ACCESS

Edited by:

Trine Bilde,

Aarhus University, Denmark

Reviewed by:

Daniel T. Blumstein,

University of California, Los Angeles,

United States

Tom Langen,

Clarkson University, United States

*Correspondence:

Tom Ratz

tom.ratz@ed.ac.uk

Specialty section:

This article was submitted to

Social Evolution,

a section of the journa

Frontiers in Ecology and Evolution

Received: 05 October 2018 Accepted: 06 November 2018 Published: 22 November 2018

Citation:

Ratz T, Castel E and Smiseth PT

(2018) Male Assistance in Parental

Care Does Not Buffer Against

Detrimental Effects of Maternal

Inbreeding on Offspring

Front. Ecol. Evol. 6:196

doi: 10.3389/fevo.2018.00196

\section{Male Assistance in Parental Care Does Not Buffer Against Detrimental Effects of Maternal Inbreeding on Offspring}

\author{
Tom Ratz*, Elise Castel and Per T. Smiseth \\ Institute of Evolutionary Biology, University of Edinburgh, Edinburgh, United Kingdom
}

The severity of inbreeding depression often varies across environments and recent work suggests that social interactions can aggravate or reduce inbreeding depression. For example, stressful interactions such as competition can exacerbate inbreeding depression, whereas benign interactions such as parental care can buffer against inbreeding depression in offspring. Here, we test whether male assistance in parental care can buffer against the detrimental effects of maternal inbreeding on offspring fitness in the burying beetle Nicrophorus vespilloides. Our results confirm that maternal inbreeding had detrimental effects on offspring survival. However, we found no evidence that male assistance in parental care buffered against those effects on offspring fitness. Outbred females benefitted from male assistance, gaining more weight over the breeding attempt when assisted by a male. In contrast, inbred females did not benefit from male assistance, gaining as much weight regardless of whether they were assisted by a male or not. Surprisingly, we find that males gained more weight during the breeding attempt when mated to an inbred female, suggesting that males benefitted from assisting an inbred female partner in terms of their weight gain. Overall, our findings suggest that parental care or other benign social interactions may not always buffer against detrimental effects of inbreeding depression.

Keywords: inbreeding depression, inbreeding-environment interaction, indirect genetic effects, Nicrophorus vespilloides, parental care

\section{INTRODUCTION}

Inbreeding depression, defined as the reduction in fitness of progeny produced as a consequence of mating between relatives, has been reported in a broad diversity of animals and plants (reviewed in Charlesworth and Charlesworth, 1987; Keller and Waller, 2002; Charlesworth and Willis, 2009). There is ample evidence for inbreeding depression in fitness-related traits, including fecundity, offspring growth and survival, and longevity, from studies conducted under both laboratory and natural conditions (Keller, 1998; Slate et al., 2000; Keller and Waller, 2002). There is growing awareness that the magnitude of inbreeding depression often varies between species or studies on the same species (e.g., Fox and Scheibly, 2006). This may reflect that inbreeding depression is often more severe under more stressful environmental conditions (Hoffmann and Parsons, 1991; Armbruster and Reed, 2005; Cheptou and Donohue, 2011). The social environment may play an 
important role in this context because social interactions can amplify or alleviate stress, thereby exacerbating or buffering against inbreeding depression. For example, direct competition between inbred and outbred males exacerbates inbreeding depression in house mice (Mus domesticus) (Meagher et al., 2000). Meanwhile, parental care buffers against inbreeding depression in offspring in the burying beetle Nicrophorus vespilloides (Pilakouta et al., 2015).

The examples provided above illustrate that social interactions with other individuals can have an important impact on the fitness of those individuals that are themselves inbred (e.g., Meagher et al., 2000; Pilakouta et al., 2015). However, there is mounting evidence for indirect genetic effects associated with inbreeding whereby outbred individuals suffer fitness costs as a result of interacting with or depending upon inbred ones (Mattey et al., 2013; Richardson and Smiseth, 2017). For example, in species where parents care for their offspring, parental inbreeding can have detrimental effects on the offspring's fitness. Recent studies on the burying beetle Nicrophorus vespilloides and red deer (Cervus elaphus) show that maternal inbreeding is associated with lower offspring survival (Mattey et al., 2013; Huisman et al., 2016). Such effects of maternal inbreeding on offspring fitness may result from inbred females providing less or lower-quality care than outbred ones (Mattey et al., 2013). Currently, it is unclear whether interactions with third-party individuals may buffer against the detrimental effects of maternal inbreeding on offspring fitness. For example, in species with biparental care, the presence of a male partner may offset some of the detrimental effects of maternal inbreeding on offspring. In support for this hypothesis, a study on zebra finches (Taeniopygia guttata) found that maternal inbreeding had no detectable effect on offspring fitness even though inbred mothers spent less time incubating their eggs (Pooley et al., 2014). In this study, males always assisted with parental care. Thus, there is now a need for studies that examine whether male assistance in parental care buffers against the detrimental effects of maternal inbreeding on offspring fitness by manipulating the presence and absence of male assistance.

The burying beetle Nicrophorus vespilloides is well-suited to test whether male assistance in care buffers against the detrimental effects of maternal inbreeding on offspring fitness. In this species, both parents cooperate to bury, maintain and guard the vertebrate carcass, which serves as the sole food source for both larvae and parents during breeding. Both parents also care for the larvae after hatching, though females spend more time provisioning food than males and males desert the brood earlier than females (Bartlett and Ashworth, 1988; Smiseth et al., 2005). Males respond to the removal or desertion of the female, and to the reduced amount of care of handicapped females, by increasing their time spent on paternal care (Smiseth et al., 2005; Royle et al., 2014; Creighton et al., 2015). Furthermore, males spend more time providing care when paired with an inbred female, suggesting that males respond to the inbreeding status of their female partner (Mattey and Smiseth, 2015). There is good evidence that maternal inbreeding has a detrimental impact on the survival of outbred offspring (Mattey et al., 2013, 2018; but see Mattey and Smiseth, 2015; Ford et al., 2018). However, there is no information as to whether male assistance in parental care buffers against the detrimental effects of maternal inbreeding on offspring fitness.

Here, we use a $2 \times 3$ factorial design to test whether male assistance in parental care buffers against the detrimental effects of maternal inbreeding on offspring fitness in the burying beetle $N$. vespilloides. We paired an inbred or outbred female with an unrelated inbred or outbred male that assisted the female with parental care during larval development. We also added additional treatments where the male was experimentally removed before larval hatching such that the inbred or outbred female received no assistance in parental care. Our first aim was to test whether male assistance in parental care buffers against the detrimental effects of maternal inbreeding on offspring fitness. If so, we predicted effects of the interaction between maternal inbreeding (inbred or outbred) and male status (inbred, outbred, or absent) on offspring fitness (i.e., mean offspring survival and/or weight), reflecting that maternal inbreeding had a greater negative impact on offspring fitness when the male was absent than when the female received assistance from a male. Furthermore, if inbred males have a reduced capacity to buffer against the detrimental effects of maternal inbreeding, we predicted that maternal inbreeding would have a greater negative impact on offspring fitness when the female was assisted by an inbred male rather than an outbred male. We next tested whether male assistance in parental care had an impact on female and male weight change whilst providing care. In this species, the amount of carrion consumed by a parent reflects parental investment in future reproduction (Creighton et al., 2009). Thus, if male assistance in parental care buffered against the detrimental effects of maternal inbreeding, thereby allowing females to save more resources for investment in future reproduction, we predicted females to gain more weight when assisted by a male than when the male was absent. If outbred males were better able to buffer for the effects of maternal inbreeding than inbred ones, we predicted that females assisted by an outbred male would gain more weight than those assisted by an inbred male. Finally, as inbred females are expected to provide lower quality care than outbred ones, we predicted that males paired with an inbred female would gain less weight than males paired with an outbred female, reflecting that the former increase their investment in current reproduction (Mattey and Smiseth, 2015).

\section{MATERIALS AND METHODS}

\section{Origin and Rearing of Experimental Beetles}

The beetles used in these experiments originated from wild beetles originally collected in Corstorphine Hill, Edinburgh, U.K. In order to avoid unintended inbreeding, we maintained a large outbred laboratory population (Mattey et al., 2018). To this end, we bred 200-300 individuals each generation, recruiting three offspring from each brood to the next generation. Nonbreeding adult beetles were kept in individual transparent plastic containers $(12 \times 8 \times 2 \mathrm{~cm})$ filled with moist soil, and fed small pieces of organic beef twice a week. The beetles were kept under constant temperature $\left(20-22^{\circ} \mathrm{C}\right)$ and photoperiod $(16: 8 \mathrm{~h}$ light:dark). 


\section{Experimental Design}

To test whether male assistance in parental care buffers against the detrimental effects of maternal inbreeding on offspring fitness, we used a $2 \times 3$ factorial design in which an inbred or an outbred female was mated with an inbred or outbred male that later assisted the female in parental care (inbred female mated to an inbred male: $N=51$; inbred female mated to an outbred male: $N=36$; outbred female mated to an inbred male: $N=48$; outbred female mated to an outbred male: $N=35$ ). Our design also included two additional treatments where an inbred or an outbred female was mated with a male that was removed before the larvae hatched $(N=40$ and $N=38$ for inbred and outbred females, respectively).

We generated inbred females and males for use as parents in this experiment by paring their mother with her fullsibling brother in the previous generation (Mattey et al., 2018). Meanwhile, we generated outbred females and males by paring their mother with an unrelated male (i.e., a male with which the mother did not share an ancestor for at least two generations; Mattey et al., 2018). Once the inbred and outbred females and males had reached sexual maturity, we randomly assigned each individual to one of the six treatments. At the start of the experiment, we weighed each female and male. We then paired inbred and outbred females with an unrelated inbred or outbred male partner depending on the treatment to which they had been assigned, and transferred them into a larger transparent plastic container $(17 \times 12 \times 6 \mathrm{~cm})$ filled with $1 \mathrm{~cm}$ of moist soil. We provided each pair with a previously frozen mouse carcass of a standardized size (22.33-26.89 g) (supplied by Livefoods Direct, Sheffield, UK) to initiate breeding. We checked each container for the presence of eggs daily and recorded the date at which the first eggs appeared as the start of egg laying. Two days after the start of egg laying, we recorded clutch size as the number of eggs visible through the bottom of the transparent containers (Monteith et al., 2012). In the limited amount of soil that we used, the number of eggs visible at the bottom of the container is strongly correlated with the actual clutch size (Monteith et al., 2012).

In those treatments where the male was absent during larval development, we removed the male from the container 2 days after the outset of egg laying as this corresponds to the day before hatching (Smiseth et al., 2006). In the remaining treatments, we left the inbred or outbred male within the container thereby allowing him to assist the female in providing parental care until the larvae completed development and dispersed from the mouse carcass (hereafter referred to as larval dispersal). At larval dispersal, we recorded brood size by counting the number of larvae in the brood and weighed the total mass of the brood. We estimated the proportion of offspring surviving until dispersal as the brood size at dispersal divided by the clutch size. We calculated mean larval mass as the total mass of the brood divided by the brood size. At larval dispersal, we also weighed each female and male parent. We then estimated the percentage of weight gain of females and males during breeding as the relative difference in body mass measured at mating $\left(W_{m}\right)$ and the body mass at larval dispersal $\left(W_{d}\right)$ using the following equation: $\frac{W_{d}-W m}{W_{m}} \times 100$. We also calculated the absolute weight gain in females and males (i.e., $\left.W_{d}-W_{m}\right)$.

\section{Statistical Analysis}

All statistical analyses were conducted using R v 3.3.3 (R Development Core Team, 2011) loaded with the package car (Fox et al., 2016). To analyze our data on offspring survival until dispersal, we used a Poisson generalized linear model. We analyzed our data on mean larval mass at dispersal and weight gain by females and males using general linear models fitted with a Gaussian error structure. These models always included maternal inbreeding (inbred or outbred) and male status (inbred, outbred, or absent) as fixed factors. We included female relative weight gain as an additional fixed factor in the model on male relative weight gain as male carrion consumption and weight change has been shown to depend on female carrion consumption and weight gain (Pilakouta et al., 2016). In the models on absolute weight gain by females and males, we also included the parent's initial mass as a fixed factor to control for potential differences in body size across individuals given that inbred females were significantly lighter than outbred females at the start of the experiment $\left(\operatorname{LR} \chi^{2}=4.43, d f=1, P=0.035\right)$. We excluded carcass size from our analyses given that we used mouse carcasses within a narrow, standardized size range (22.33$26.89 \mathrm{~g}$ ) in our experiment. Furthermore, there was no significant effect of carcass size in any of our analyses when we included it as a fixed factor. We assessed and evaluated whether the structure of all models was appropriate for each variable by plotting the residuals from the models. Whenever there was a significant effect of male status (inbred, outbred or, absent), we tested for differences between each treatment using Tukey contrasts reporting $p$-values based on the Bonferroni correction for multiple testing. The complete dataset and $\mathrm{R}$ code used for the analyses are provided in Data Sheet 1 and Data Sheet 2 (Supplementary Material).

\section{RESULTS}

\section{Offspring Fitness}

There was no significant effect of the interaction between maternal inbreeding (inbred or outbred) and male status (inbred, outbred, or absent) on the proportion of offspring surviving until dispersal (Maternal inbreeding:Male status, Table 1, Figure 1A). Thus, there was no evidence that male assistance in parental care buffered against the detrimental effects of maternal inbreeding on offspring fitness.

We next explored potential main effects of maternal inbreeding and male status on offspring fitness. As expected, broods reared by outbred females had a larger proportion of offspring surviving until dispersal than broods reared by inbred females (Table 1 and Figure 1A), thus confirming that there were detrimental effects of maternal inbreeding on offspring survival. There was no difference in the proportion of offspring surviving until dispersal depending on whether the male was inbred, outbred or absent (Table 1, Figure 1A). There was no difference in mean larval mass at dispersal between inbred and outbred females (Table 1 and Figure 1B). Likewise, there were 
TABLE 1 | Effects of maternal inbreeding (inbred or outbred) and male status (inbred, outbred or absent) on offspring survival until dispersal and mean larval mass at larval dispersal.

\begin{tabular}{llllllllll}
\hline & \multicolumn{3}{c}{ Offspring survival } & & \multicolumn{3}{c}{ Mean larval mass } \\
\cline { 2 - 3 } & LR $\chi^{2}$ & df & $\boldsymbol{P}$ & & LR $\chi^{2}$ & df & $\boldsymbol{P}$ \\
\hline Maternal inbreeding:Male status & 0.275 & 2 & 0.871 & & 2.85 & 2 & 0.239 \\
Maternal inbreeding & 9.55 & 1 & $\mathbf{0 . 0 0 2}$ & & 1.21 & 1 & 0.270 \\
Male status & 0.711 & 2 & 0.700 & & 3.52 & 2 & 0.172 \\
\hline
\end{tabular}

Values are obtained from GLMs.

$L R$, likelihood ratio. Statistically significant $P$ values $(<0.05)$ are shown in boldface.

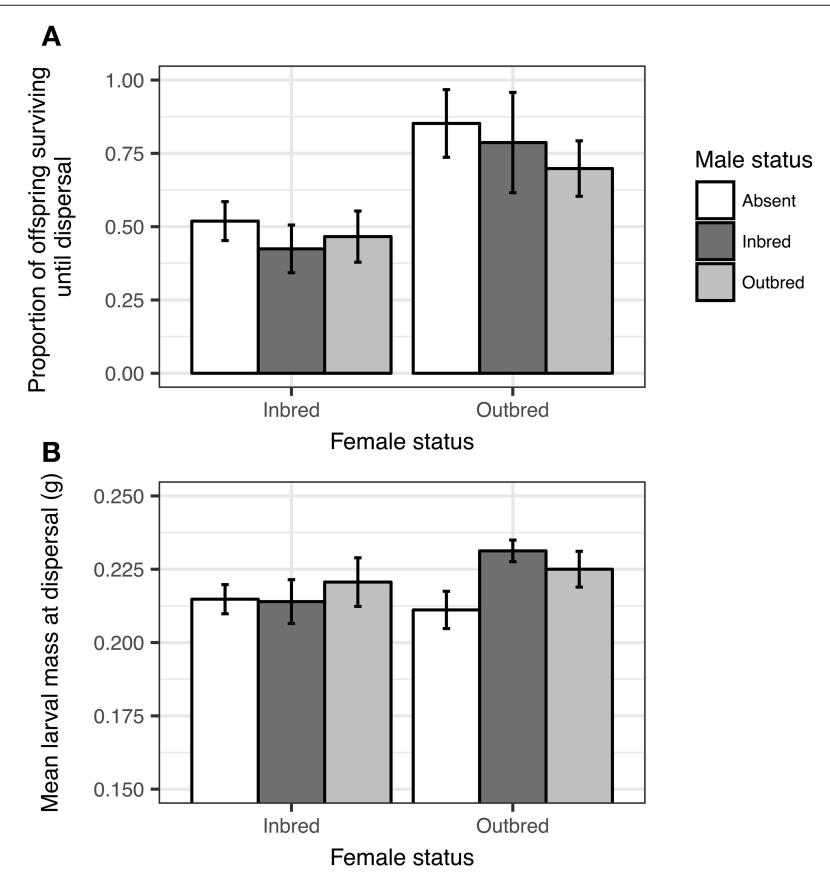

FIGURE 1 | Effects of maternal inbreeding (inbred or outbred) and male status (inbred, outbred, or absent) on the proportion of offspring surviving until dispersal (A), and on mean larval mass at dispersal (B). Mean $\pm \mathrm{SE}$.

no significant main effects of male status on mean larval mass at dispersal (Table 1 and Figure 1B). These findings suggest that there was no detrimental effect of maternal or paternal inbreeding on mean offspring weight and that male assistance had no positive main effects on mean offspring weight.

\section{Female and Male Weight Gain}

There was a significant effect of the interaction between maternal inbreeding and male status on both relative and absolute weight gain of females (Table 2). This interaction effect reflected that outbred females gained more weight when a male assisted in parental care than when the male was removed (Figure 2A), while inbred females gained a similar amount of mass regardless of whether the male assisted with parental care or not. There was no difference in either relative or absolute weight gain of females depending on whether the male was inbred or outbred (Female inbred; Male outbred vs. Male inbred: Estimate $=-0.010 \pm$
0.007, $Z=-1.34, P>0.999$; Female outbred; Male outbred vs. Male inbred: Estimate $=0.007 \pm 0.008, Z=0.891, P>0.999$ ) Thus, there was no evidence that females gained more weight when assisted by an outbred rather than an inbred male.

There was no significant effect of the interaction between maternal inbreeding and male status on either relative or absolute weight gain of males (Table 2 and Figure 2B). There were no significant differences in either relative or absolute weight gain between inbred and outbred males (Table 2 and Figure 2A). However, males gained less relative and less absolute weight when paired with an outbred female than when paired with an inbred female (Figure 2A), suggesting that males benefitted from having an inbred partner.

\section{DISCUSSION}

Here, we tested whether male assistance in parental care buffers against the detrimental effects of maternal inbreeding on offspring fitness in $N$. vespilloides. We found that maternal inbreeding had detrimental effects on offspring fitness in terms of reduced offspring survival, confirming the results of prior work on this species (Mattey et al., 2013, 2018; but see Mattey and Smiseth, 2015; Ford et al., 2018) and consistent with evidence from studies on vertebrate systems (Keller, 1998; Bérénos et al., 2016; Huisman et al., 2016). However, we found no evidence that male assistance in parental care buffered against these detrimental effects. Male assistance in care had a positive effect on female weight gain during breeding, showing that male assistance was beneficial to females. However, this was only the case when females were outbred, suggesting that outbred females benefitted more from male assistance than inbred ones. Finally, males paired with an inbred female gained more weight than those paired with an outbred female. This finding is opposite to what we predicted if males paired with an inbred female increased their investment in current reproduction. Instead, this result may reflect that males paired with an inbred female spent more time provisioning food to the larvae, thereby gaining better access to consume food from the carcass (Pilakouta et al., 2016). Overall, our results provide no evidence that male assistance in parental care buffers against the detrimental effects of maternal inbreeding on offspring fitness. Below, we provide a more detailed discussion of our results and their implications for our understanding of the consequences of inbreeding in populations where social interactions are prevalent.

Our first key finding was that maternal inbreeding had detrimental effects on offspring fitness in terms of reduced larval survival from egg laying until dispersal, but that this effect was independent of whether the male was absent or present, and when the male was present, whether the male was inbred or outbred. Thus, our results provide no evidence that male assistance in parental care buffered against the detrimental effects of maternal inbreeding on offspring fitness. This finding contrasts with experimental evidence from a recent study on zebra finches, suggesting that male assistance in parental care buffers against the detrimental effects of maternal inbreeding. In zebra finches, inbred females spend less time incubating eggs, and buffering 
TABLE 2 | Effects of maternal inbreeding (inbred or outbred) and male status (inbred, outbred or absent) on female and male relative and absolute weight gains over the breeding attempt.

\begin{tabular}{|c|c|c|c|c|c|c|c|c|c|c|c|c|}
\hline & \multicolumn{3}{|c|}{ Female relative weight gain } & \multicolumn{3}{|c|}{$\begin{array}{l}\text { Female absolute weight } \\
\text { gain }\end{array}$} & \multicolumn{3}{|c|}{ Male relative weight gain } & \multicolumn{3}{|c|}{ Male absolute weight gain } \\
\hline & $\operatorname{LR} \chi^{2}$ & df & $\boldsymbol{P}$ & $\operatorname{LR} \chi^{2}$ & df & $P$ & $\operatorname{LR} \chi^{2}$ & df & $\boldsymbol{P}$ & $\operatorname{LR} \chi^{2}$ & df & $P$ \\
\hline Maternal inbreeding:Male status & 6.59 & 2 & 0.037 & 7.80 & 2 & 0.020 & 0.067 & 1 & 0.795 & 0.064 & 1 & 0.799 \\
\hline Maternal inbreeding & 0.737 & 1 & 0.390 & 1.61 & 1 & 0.203 & 5.11 & 1 & 0.023 & 3.91 & 1 & 0.047 \\
\hline Male status & 2.16 & 2 & 0.339 & 3.46 & 2 & 0.176 & 0.131 & 1 & 0.717 & 0.039 & 1 & 0.842 \\
\hline Parent's initial weight & - & - & - & 0.006 & 1 & 0.938 & - & - & - & 1.26 & 1 & 0.261 \\
\hline Female relative weight gain & - & - & - & - & - & - & 2.31 & 1 & 0.127 & - & - & - \\
\hline Female absolute weight gain & - & - & - & - & - & - & - & - & - & 2.31 & 1 & 0.127 \\
\hline
\end{tabular}

Values are obtained from GLMs.

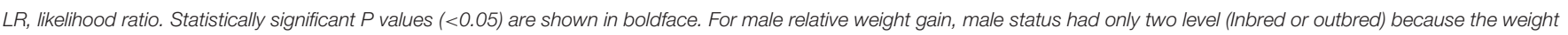
was not recorded for males of the "absent" treatment group.

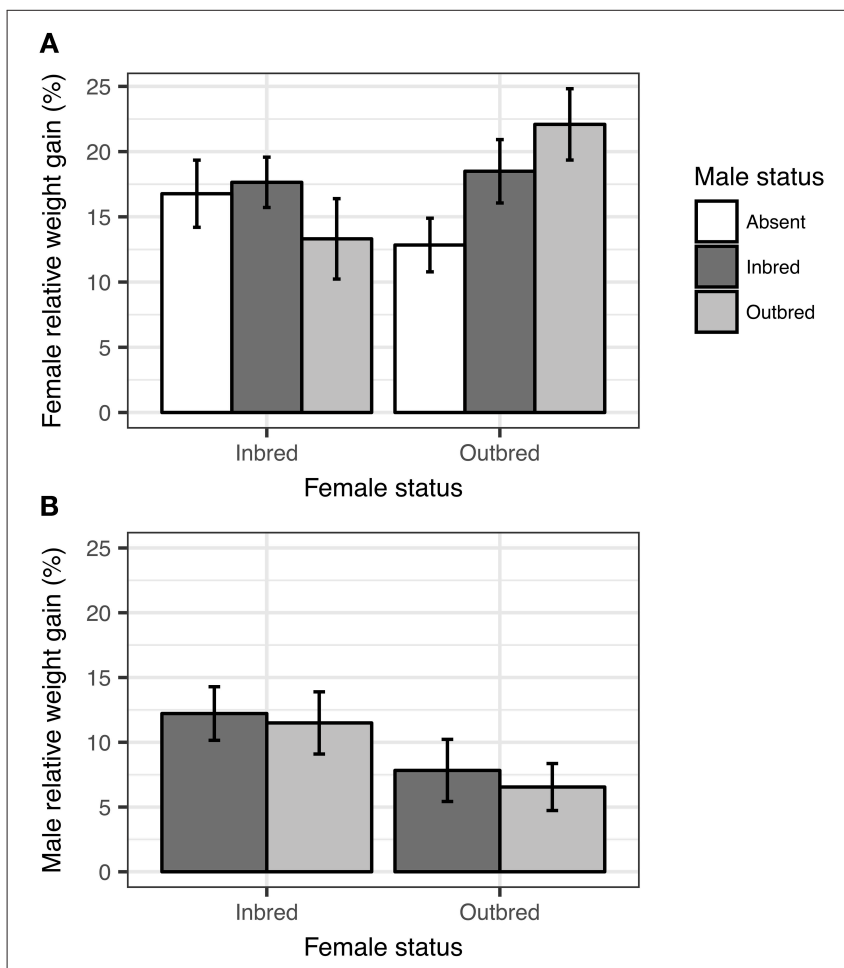

FIGURE 2 | Effects of maternal inbreeding (inbred or outbred) and male status (inbred, outbred, or absent) on female relative weight gain (A), and male relative weight gain $\mathbf{( B )}$ over the breeding attempt. Mean $\pm \mathrm{SE}$.

is thought to reflect that males compensate for the reduced incubation by inbred females (Pooley et al., 2014). Prior work on $N$. vespilloides shows that maternal inbreeding can reduce offspring fitness both before hatching (i.e., hatching success of eggs; Mattey and Smiseth, 2015; Ford et al., 2018) and after (i.e., survival at the larval stage; Mattey et al., 2013, 2018). Thus, one potential explanation for why male assistance did not buffer against the detrimental effects of maternal inbreeding on offspring is that post-hatching male care cannot buffer against effects on hatching success of eggs. This explanation may also apply to other systems as detrimental effects of maternal inbreeding on hatching success have also been reported for example in song sparrows (Keller, 1998). An alternative explanation for why male assistance in parental care did not buffer against the detrimental effects of maternal inbreeding is that male assistance in care in this species does not increase larval survival from hatching until dispersal under laboratory conditions (Smiseth et al., 2005). This presumably reflects that males contribute far less toward parental care than females in this species (Smiseth et al., 2005). This sex difference in parental care may also explain why there were detrimental effects of maternal inbreeding on offspring fitness, whilst there were no detrimental effects of paternal inbreeding. We note that male assistance in guarding and defending the brood against predators or conspecific intruders plays an important role under natural conditions in burying beetles (Scott, 1990). Thus, it is possible that male assistance in parental care could buffer against detrimental effects of maternal inbreeding on offspring fitness under natural conditions where competitors or conspecific intruders may reduce offspring survival. Further studies are now needed to investigate whether male assistance in care might buffer against detrimental effects of maternal inbreeding when there is a risk of predation or takeovers by conspecific intruders.

Our second main finding was that there was an effect of the interaction between maternal inbreeding and male status on female weight gain with male assistance in parental care having a positive effect on relative mass gain of outbred females only. We anticipated that females would benefit from male assistance regardless of their own inbreeding status, and that this would lead to an increase in their mass gain indicative of a shift toward investment in future reproduction (Creighton et al., 2009). Thus, this finding suggests that outbred females benefitted more from male assistance than inbred ones. One potential explanation for why this might be the case is that inbreeding is associated with terminal investment and that inbred females therefore always invest more effort into current reproduction. There is some evidence that inbreeding is associated with terminal investment from previous studies on N. vespilloides (Mattey and Smiseth, 2015; Richardson and Smiseth, 2017; Mattey et al., 2018). In light 
of our finding that the presence of a male had a positive effect on the weight gain of outbred females only, future work should now test for a differential effect of male assistance in care on the subsequence breeding performance by outbred and inbred females. Presumably, outbred females would perform better in subsequent breeding attempts when assisted by a male during a first breeding attempt, while inbred females would perform equally well regardless of whether they were assisted by a male or not.

We found no evidence that females gained more mass when assisted by an outbred male, suggesting that females did not benefit more from assistance by outbred males as compared to inbred ones. We predicted that females would gain more mass when assisted by an outbred male if outbred males are better parents than inbred ones. Our results show that that this was not case, which might explain why outbred males were not better able to buffer against the detrimental effects of maternal inbreeding on offspring fitness. Our finding echoes previous work in this species showing that characteristics of the male, such as body size, have little influence on carrion consumption or weight gain of the female, whereas females adjust their consumption and weight gain to match their male partner's weight gain (Pilakouta et al., 2016). In light of this evidence, and keeping in mind that there was no difference in weight gain by inbred and outbred males in our experiment (see discussion below), it seems unlikely that females adjusted their carrion consumption and weight change to the status of their male partner.

The final main result of our study was that males paired with inbred females gained more weight over the breeding attempt than males paired with outbred females. We predicted that males paired with inbred females would gain less weight over the breeding attempt. The reason for this is that males paired with inbred females should be expected to increase their investment into current reproduction to compensate for the detrimental effects of maternal inbreeding. Thus, our finding suggests that males instead might increase their investment into future reproduction when their partner is inbred. However, this seems unlikely given that a previous study on $N$. vespilloides found that males paired with inbred females provided more care than males paired with outbred females (Mattey and Smiseth, 2015). An alternative explanation is that males paired with an inbred female gained more weight over the breeding attempt because they provided more care than males paired with an outbred female. In this species, parents feed from the carcass whilst breeding and males might gain better access to the carcass if they provide more care (Pilakouta et al., 2016). If so, we might expect a positive correlation between male food provisioning and male weight gain in this species. Altogether, our findings suggest that males benefitted in terms of gaining more weight during the breeding attempt when assisting an inbred partner. Given that male weight gain serves as a proxy for investment in future reproduction (Creighton et al., 2009), one avenue for future work is to compare the subsequent reproductive performance of males paired with an outbred or inbred female during a previous breeding attempt.
Our results have broader implications for understanding how social interactions shape the severity of inbreeding depression. There is increasing evidence that social interactions can alter the severity of inbreeding depression, with stressful interactions aggravating the severity of inbreeding depression (e.g., Meagher et al., 2000) and benign interactions buffering against inbreeding depression (e.g., Pilakouta et al., 2015). It is well documented that maternal care enhances larval survival and growth in burying beetle (e.g., Eggert et al., 1998; Trumbo, 2007; Arce et al., 2012). Thus, maternal care may buffer against inbreeding depression in offspring by reducing environmental stresses to offspring, such as the risk of death due to starvation, infanticide by conspecific intruders, and predation. In contrast, as discussed above, there is mixed evidence as to whether male assistance in care enhances offspring fitness (Pooley et al., 2014; our study). Thus, our results suggest that parental care or other benign social interactions will not always buffer against inbreeding depression. There is now a need for further work on the buffering effects of male assistance in parental care against the detrimental effects of maternal inbreeding on offspring fitness in systems where males contribute more toward care. For example, such experiments could be conducted on bird species where males and females contribute more equally toward parental care (Clutton-Brock, 1991).

\section{ETHICS STATEMENT}

Our study adheres to the Guidelines for the Use of Animals in Research, the legal requirements of the U.K., as well as all institutional guidelines at The University of Edinburgh. None of the procedures used in this study had the potential to cause pain or distress. To minimize disturbance during breeding, the beetles were only handled at mating and dispersal.

\section{AUTHOR CONTRIBUTIONS}

TR and PS conceived and designed the experiments. TR and EC collected the data. TR analyzed the data. TR and PS wrote the first draft of the manuscript, with contributions from all authors.

\section{FUNDING}

TR was supported by the Darwin Trust of Edinburgh.

\section{ACKNOWLEDGMENTS}

We thank the City of Edinburgh Natural Heritage Service for permission to collect beetles in their reserve at Corstorphine Hill. We also thank Jon Richardson and Pauline Comin for assistance with maintaining the laboratory population.

\section{SUPPLEMENTARY MATERIAL}

The Supplementary Material for this article can be found online at: https://www.frontiersin.org/articles/10.3389/fevo. 2018.00196/full\#supplementary-material 


\section{REFERENCES}

Arce, A. N., Johnston, P. R., Smiseth, P. T., and Rozen, D. E. (2012). Mechanisms and fitness effects of antibacterial defences in a carrion beetle. J. Evol. Biol. 25, 930-937. doi: 10.1111/j.1420-9101.2012.02486.x

Armbruster, P., and Reed, D. H. (2005). Inbreeding depression in benign and stressful environments. Heredity 95, 235-242. doi: 10.1038/sj.hdy.6800721

Bartlett, J., and Ashworth, C. M. (1988). Brood size and fitness in Nicrophorus vespilloides (Coleoptera, Silphidae). Behav. Ecol. Sociobiol. 22, 429-434. doi: 10.1007/BF00294981

Bérénos, C., Ellis, P. A., Pilkington, J. G., and Pemberton, J. M. (2016). Genomic analysis reveals depression due to both individual and maternal inbreeding in a free-living mammal population. Mol. Ecol. 25, 3152-3168. doi: $10.1111 / \mathrm{mec} .13681$

Charlesworth, D., and Charlesworth, B. (1987). Inbreeding depression and its evolutionary consequences. Annu. Rev. Ecol. Syst. 18, 237-268. doi: 10.1146/annurev.es.18.110187.001321

Charlesworth, D., and Willis, J. H. (2009). The genetics of inbreeding depression. Annu. Rev. Ecol. Syst. 10, 783-796. doi: 10.1038/nrg2664

Cheptou, P. O., and Donohue, K. (2011). Environment-dependent inbreeding depression: its ecological and evolutionary significance. New Phytol. 189, 395-407. doi: 10.1111/j.1469-8137.2010.03541.x

Clutton-Brock, T. H. (1991). The Evolution of Parental Care. Princeton, NJ: Princeton University Press.

Creighton, C. J., Smith, A. N., Komendat, A., and Belk, M. C. (2015). Dynamics of biparental care in a burying beetle: experimental handicapping results in partner compensation. Behav. Ecol. Sociobiol. 69, 265-271. doi: $10.1007 /$ s00265-014-1839-z

Creighton, J. C., Heflin, N. D., and Belk, M. C. (2009). Cost of reproduction, resource quality, and terminal investment in a burying beetle. Am. Nat. 174, 673-684. doi: 10.1086/605963

Eggert, A. K., Reinking, M., and Muller, J. K. (1998). Parental care improves offspring survival and growth in burying beetles. Anim. Behav. 55, 97-107. doi: 10.1006/anbe.1997.0588

Ford, L. E., Henderson, K. J., and Smiseth, P. T. (2018). Differential effects of offspring and maternal inbreeding on egg laying and offspring performance in the burying beetle Nicrophorus vespilloides. J. Evol. Biol. 31, 1047-1057. doi: $10.1111 /$ jeb. 13285

Fox, C. W., and Scheibly, K. L. (2006). Variation in inbreeding depression among populations of seed beetle, Stator limbatus. Entomol. Exp. Appl. 121, 137-144. doi: 10.1111/j.1570-8703.2006.00465.x

Fox, J., Weisberg, S., Bates, D., and Fox, M. J. (2016). Package “Car.” Avaliable online at: http://cran.r-project.org/web/packages/car/car.pdf

Hoffmann, A. A., and Parsons, P. A. (1991). Evolutionary Genetics and Environmental Stress. Oxford: Oxford University Press.

Huisman, J., Kruuk, L. E. B., Ellis, P. A., Clutton-Brock, T. H., and Pemberton, J. M. (2016). Inbreeding depression across the lifespan in a wild mammal population. Proc. Natl. Acad. Sci. U. S. A. 113, 3585-3590. doi: 10.1073/pnas.15180 46113

Keller, L. F. (1998). Inbreeding and its fitness effects in an insular population of song sparrows (Melospiza melodia). Evolution 52, 240-250.

Keller, L. F., and Waller, D. M. (2002). Inbreeding effects in wild populations. Trends Ecol. Evol. 17, 230-241. doi: 10.1016/S0169-5347(02)02489-8

Mattey, S. N., Richardson, J., Ratz, T., and Smiseth, P. T. (2018). Effects of offspring and parental inbreeding on parent-offspring communication. Am. Nat. 191, 716-725. doi: 10.1086/697236
Mattey, S. N., and Smiseth, P. T. (2015). Complex effects of inbreeding on biparental cooperation. Am. Nat. 185, 1-12. doi: 10.1086/679067

Mattey, S. N., Strutt, L., and Smiseth, P. T. (2013). Intergenerational effects of inbreeding in Nicrophorus vespilloides: Offspring suffer fitness costs when either they or their parents are inbred. J. Evol. Biol. 26, 843-853. doi: $10.1111 /$ jeb.12102

Meagher, S., Penn, D. J., and Potts, W. K. (2000). Male-male competition magnifies inbreeding depression in wild house mice. Proc. Natl. Acad. Sci. U. S. A. 97, 3324-3329. doi: 10.1073/pnas.97.7.3324

Monteith, K. M., Andrews, C., and Smiseth, P. T. (2012). Post-hatching parental care masks the effects of egg size on offspring fitness: a removal experiment on burying beetles. J. Evol. Biol. 25, 1815-1822. doi: 10.1111/j.1420-9101.2012.02567.x

Pilakouta, N., Jamieson, S., Moorad, J. A., and Smiseth, P. T. (2015). Parental care buffers against inbreeding depression in burying beetles. Proc. Natl. Acad. Sci. U. S. A. 112, 8031-8035. doi: 10.1073/pnas.1500658112

Pilakouta, N., Richardson, J., and Smiseth, P. T. (2016). If you eat, i eat: resolution of sexual conflict over consumption from a shared resource. Anim. Behav. 111 175-180. doi: 10.1016/j.anbehav.2015.10.016

Pooley, E. L., Kennedy, M. W., and Nager, R. G. (2014). Maternal inbreeding reduces parental care in the zebra finch, Taeniopygia guttata. Anim. Behav. 97, 153-163. doi: 10.1016/j.anbehav.2014.09.012

R Development Core Team (2011). R: A Language and Environment for Statistical Computing. Vienna: The R Foundation for Statistical Computing. Avaliable online at: http://www.R-project.org/

Richardson, J., and Smiseth, P. T. (2017). Intraspecific competition and inbreeding depression: increased competitive effort by inbred males is costly to outbred opponents. Am. Nat. 189, 539-548. doi: 10.1086/691328

Royle, N. J., Russell, A. F., and Wilson, A. J. (2014). The evolution of flexible parenting. Science 345, 776-781. doi: 10.1126/science.1253294

Scott, M. P. (1990). Brood guarding and the evolution of male parental care in burying beetles. Behav. Ecol. Sociobiol. 26, 31-39 doi: 10.1007/BF00174022

Slate, J., Kruuk, L. E., Marshall, T. C., Pemberton, J. M., and Clutton-Brock, T. H. (2000). Inbreeding depression influences lifetime breeding success in a wild population of red deer (Cervus elaphus). Proc. R. Soc. B Biol. Sci. 267, 1657-1662. doi: 10.1098/rspb.2000.1192

Smiseth, P. T., Dawson, C., Varley, E., and Moore, A. J. (2005). How do caring parents respond to mate loss? Differential response by males and females. Anim. Behav. 69, 551-559. doi: 10.1016/j.anbehav.2004.06.004

Smiseth, P. T., Ward, R. J. S., and Moore, A. J. (2006). Asynchronous hatching in Nicrophorus vespilloides, an insect in which parents provide food for their offspring. Funct. Ecol. 20, 151-156. doi: 10.1111/j.1365-2435.2006.01072.x

Trumbo, S. T. (2007). Defending young biparentally: female risk-taking with and without a male in the burying beetle, Nicrophorus pustulatus. Behav. Ecol. Sociobiol. 61, 1717-1723. doi: 10.1007/s00265-007-0403-5

Conflict of Interest Statement: The authors declare that the research was conducted in the absence of any commercial or financial relationships that could be construed as a potential conflict of interest.

Copyright (C) 2018 Ratz, Castel and Smiseth. This is an open-access article distributed under the terms of the Creative Commons Attribution License (CC BY). The use, distribution or reproduction in other forums is permitted, provided the original author(s) and the copyright owner(s) are credited and that the original publication in this journal is cited, in accordance with accepted academic practice. No use, distribution or reproduction is permitted which does not comply with these terms. 